\title{
CARDIOVASCULAR \\ MAGNETIC \\ RESONANCE \\ What cardiologists need to know about cardiovascular magnetic resonance (CMR)
}

\section{J. Cameron Bridgman*, Govind Srinivasan"\# and \\ Joseph B. Selvanayagam*\#}

*Department of Cardiology, Flinders Medical Centre, Adelaide, Australia "Department of Medicine, Flinders University of South Australia,

Adelaide, Australia

Address for correspondence:

Joseph B. Selvanayagam

Department of Medicine

Flinders University of South Australia

Adelaide

5042

Australia

Email:

joseph.selvanayagam@flinders.edu.au

\section{INTRODUCTION}

Although considered by many to be a novel imaging technique CMR has been in clinical use for over 15 years and is part of routine cardiology practice in many countries. The "novelty" seems to come from lack of availability and therefore experience with the modality. This article aims to provide an explanation of how clinical cardiac MRI fits into the diagnostic armoury of the clinical cardiologist.

The commonly expressed benefits of CMR include terms such as "non-invasive", "non-ionising" and "safe"; however there are much more robust reasons to be mentioned. CMR often makes the diagnosis where other tests would fail mainly due its unique ability to characterise myocardial tissue but also due to the clarity of functional imaging.(I) MR has significantly advanced our understanding of cardiac pathology and yielded new insights into management and prognosis.

\section{HOW A CMR IS EXECUTED}

CMR not only requires a magnet with appropriate cardiac software, but highly trained technicians, cardiologists and radiologists to

\section{ABSTRACT}

Cardiovascular Magnetic Resonance (CMR) is increasingly used in the evaluation of patients with cardiac and aortic disease. The ability to characterise myocardial tissue, function and anatomy (in any plane) without any exposure to ionising radiation are the main advantages over other imaging modalities used in cardiology. In this article we discuss the principles underlying the imaging technique, safety issues, indications and strengths of CMR. It aims to provide a concise, practical overview for the general cardiologist. SAHeart 2012; 9:82-88

acquire and interpret the scan. CMR is an interactive process that doesn't just require the operator to simply "push a button".

Clinical scans are acquired on a I.5 or 3 Tesla machine. Surprisingly the stronger field strength does not always provide superior cardiac imaging due to a number of specific artifacts at 3T.(2) Most scans generally take 20 - 40 minutes to complete although more complex cases such as adult congenital imaging can take up to I hour. The patient therefore has to be able to tolerate lying supine, generally, for this period of time without a break - which can be problematic in patients with decompensated heart failure.

Images are acquired during breath-holds of 10 - 15 seconds. The image is formed from data collected over consecutive heartbeats and mis-registration occurs if the heart is moving with respiration. Similar artifacts occur with arrhythmias which can render a scan non-diagnostic. Controlled atrial fibrillation and low frequency ectopy are manageable, high frequency ectopy can however cause major problems. Pharmacological control of the arrhythmia should be considered prior to imaging (e.g. beta blockers).

Anatomic spin-echo images are acquired first in the traditional anatomic planes. Standard sets of functional (cine) images are then acquired following prescribed rules. These include vertical and horizontal long axis (2chamber/4ch), LVOT and a short axis stack. Additional cines are acquired as needed which can include 
dedicated images of the right heart, pulmonary arteries and aorta. Tissue characterisation images are subsequently acquired in the same planes. These can include sequences to highlight water and thus tissue oedema (T2 weighted-STIR images), myocardial iron (T2*) and focal fibrosis (late gadolinium, inversion recovery imaging) as needed. Following the scan, the short axis stack is analysed with semi-automated, border detection software to give highly reproducible measurements of ventricular volumes and function. ${ }^{(3-6)}$ Chelated gadolinium-based contrast agents are used to characterise the myocardium and often for MR angiography. These agents do not contain iodinated material and are not contra-indicated in patients with previous reactions to radio-opaque contrast agents. Gadolinium-based contrast agents are generally well tolerated with I-5\% patients experiencing minor nausea and transient headache. Allergic reactions are rare and are reported to occur in every 10000 - 50000 patients. A number of cases of nephrogenic systemic fibrosis (NSF) have now been reported in patients with end-stage renal failure receiving linear gadolinium agents. ${ }^{(7-9)}$ NSF is much less likely with the newer cyclical compounds however, gadolinium administration is relatively contra-indicated in patients with impaired renal function and should be considered contraindicated in patients with severe kidney disease. That said, it is generally considered reasonable to administer cyclical gadolinium agents in patients with a GFR greater than $30 \mathrm{ml} / \mathrm{min}$ if clinically indicated. Dialysis has not yet been proven to prevent NSF but can aid clearance of Gd-DTPA. ${ }^{(9)}$

Gadolinium is taken up from the blood pool into the interstitium of tissue. The myocardial interstitial space is increased in areas of inflammation, infiltration and fibrosis. Gadolinium alters the TI relaxation time of these areas thereby altering the signal obtained relative to the normal myocardium. These areas appear white (hyper-enhanced) as opposed to normal black (nulled) myocardium. Different patterns of hyper-enhancement suggest different pathologies; for instance, myocardial infarction is seen beginning from the sub-endocardium however the distribution of hyper-enhancement in myocarditis is mid-wall or sub-epicardial. ${ }^{(10)}$

\section{SAFETY AND CONTRA-INDICATIONS}

As a MR system consists of a large, static magnetic field in which radiofrequency (RF) energy is periodically released during imaging, there are possible hazards associated with its use. In general, the potential hazard of implants or devices is dependent on factors such as its degree of ferromagnetism, geometry, location in the body as well as the gradient and field strength of the imaging magnet. In assessing whether a particular patient should be subjected to a CMR scan, the main rule, as with other investigations in medicine, is to determine the risk-benefit ratio to the patient of the proposed study.

It is absolutely essential to carefully interview the patient prior to a MRI examination, and most centres would require a safety questionnaire to be completed. Whenever there is a concern about the safety of a patient with an implant, the CMR examination should be deferred until the device and the issues associated with it are clarified. Reference texts [Shellock FG. Guide to MR Procedures and Metallic Objects: Update 200I, 7th edition. Philadelphia: Lippincott, Williams and Wilkins Healthcare, 200I] and web-based information (www.MRIsafety.com) on the safety of specific devices are available.

Pacemakers remain a contra-indication to cardiac MR currently, however most loop recorders are considered safe at I.5T. Patients with prosthetic heart valves and coronary stents (as early as 24 hour after implantation) can be safely imaged at 1.5T.(11,12) Some image degradation can occur with prosthetic material due to local disruption of the magnetic field.

Most, if not all orthopaedic implants, are safe. However, loose, metallic foreign bodies, cochlear implants and intracranial aneurysm clips are considered a strong contra-indication to MRI.

Newer MRI machines have larger bores however, claustrophobia can still be a problem. Minor degrees of anxiety can be overcome utilising blindfolds, prism spectacles or scanning prone. Sedation is generally avoided as patient cooperation is required although general anaesthesia can be used in specific circumstances.

\section{WHEN AND WHY SHOULD CMR BE CONDUCTED?}

The incremental value of CMR above echocardiography lies in the ability to characterise myocardial tissue and image the heart unencumbered by other thoracic structures eg ribs, lungs. Prescribed imaging plans eliminate foreshortening and ensure accurate 


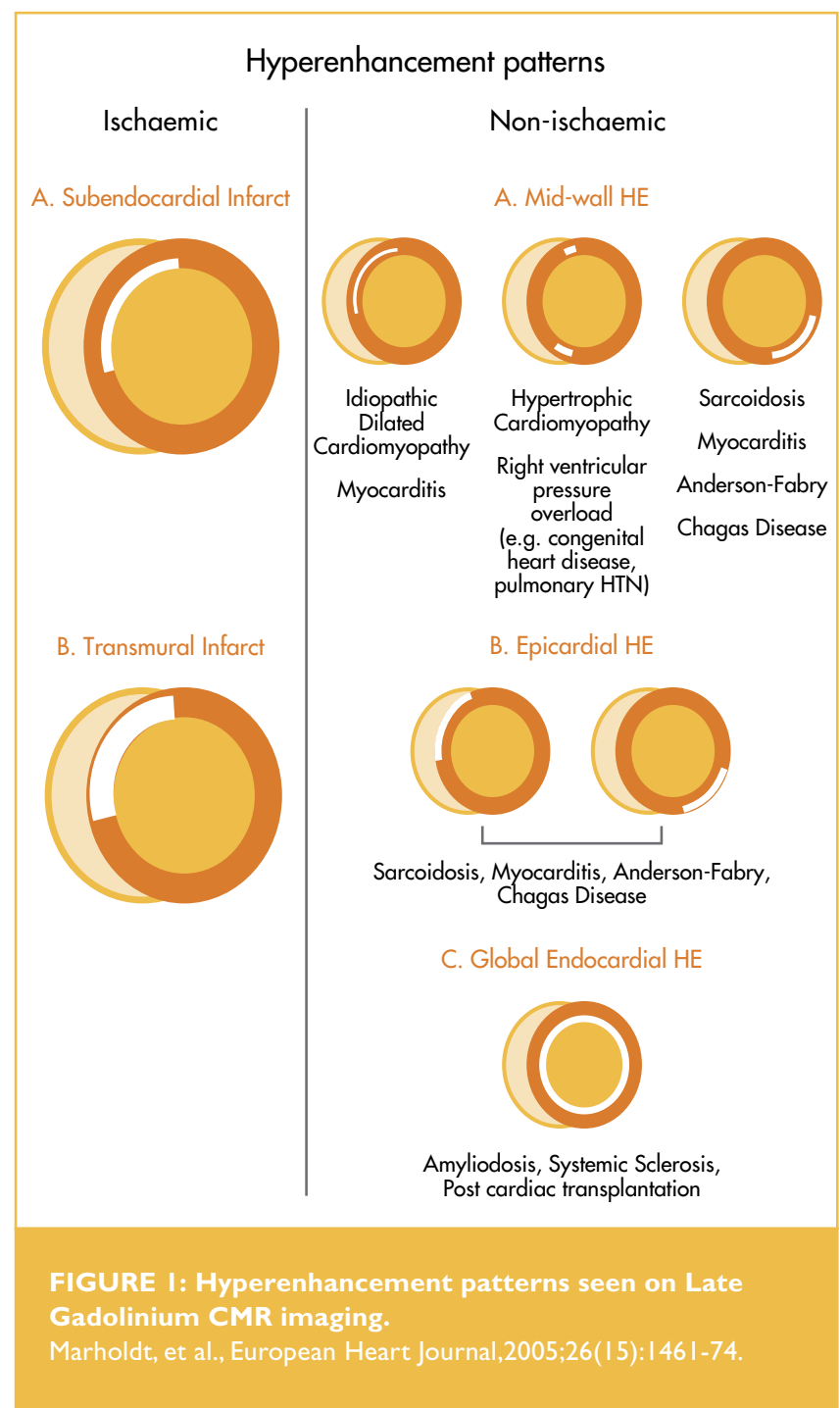

cross-sectional measurement. The ability to clearly image the apex is obviously important when looking for mural thrombus or apical hypertrophic cardiomyopathy.(13) Although, a detailed review of each CMR indication is outside the scope of this article, the major current clinical uses are described in brief.

\section{Tissue characterisation in heart failure}

A major indication for CMR is to clarify the aetiology of heart failure. The formerly used gadolinium enhancement CMR technique (LGE-CMR) has potential roles in both diagnosis and prognosis of newly diagnosed heart failure patients. Specific patterns of fibrosis and scarring have been identified in many of the cardiomyopathy states ${ }^{(14,15)}$ and are summarised in Figure I. Ischaemic cardiomyopathy is characterised by sub-endocardial-based areas of late enhancement that correlate to irreversible myocardial necrosis on histopathology (Figure 2), a pattern consistent with the "wave front phenomenon" as described by Reimer and colleagues. ${ }^{(16)}$ Patients who have non-ischaemic dilated cardiomyopathy may also have LGE-CMR evidence of scarring in up to $30 \%$ of cases; however, this is typically in a non-coronary distribution and frequently appears as a mid-wall striate. ${ }^{(14)}$ Therefore, based on the presence and pattern of myocardial fibrosis, the aetiology of the cardiomyopathy can be accurately ascertained. Delineation of the underlying aetiology is of clinical value for patients with heart failure. In patients with ischaemic substrate causing their heart failure, the delineation of potential areas of myocardial ischaemia and viability is crucial to defining clinical management. In nonischaemic cardiomyopathy, extensive mid-wall fibrosis at presentation of symptoms, is indicative of a worse prognosis and a poorer response to standard heart failure therapy. ${ }^{(17)}$ Other conditions such as cardiac amyloidosis, sarcoidosis and endomyocardial fibrosis all have classical patterns of late gadolinium hyperenhancement (see Figure 3).

Left ventricular hypertrophy (LVH) diagnosed by ECG, echo or chest radiography can be formally assessed by CMR especially in the absence of an obvious cause such as hypertension or obstructive aortic valve disease. A number of conditions can masquerade as phenocopies of hypertensive LVH including hypertrophic cardiomyopathy, amyloidosis and cardiac tumours.

\section{Myocardial viability}

Two CMR techniques are currently used for the assessment of myocardial viability:

- Late Gadolinium Enhancement CMR (LGE-CMR), a technique unique to CMR, that defines the transmural extent of scar, and

- Dobutamine CMR analogous to Dobutamine echocardiography that measures the contractile reserve of dysfunctional myocardium and is interpreted by visual analysis.

In the setting of chronic ischaemic cardiomyopathy, a number of clinical studies have examined whether the transmurality of the myocardial infarction can predict recovery of contractile function. Essentially, these studies found that the likelihood of improvement in regional function after revascularisation decreased progressively as the transmural extent of LGE before revascularisation increased. ${ }^{(18)}$ This assessment is generally used to guide revascu- 
larisation, but can also be used to plan resynchronisation therapy ("dead meat don't beat" i.e. one cannot pace non-viable scarred myocardium).
One of the key determinants of prognosis in ischaemic LV dysfunction is left ventricular end-diastolic volume and ejection fraction (EF). Kim, et al. utilising LGE-CMR, ${ }^{(19)}$ and Rizello, et al.
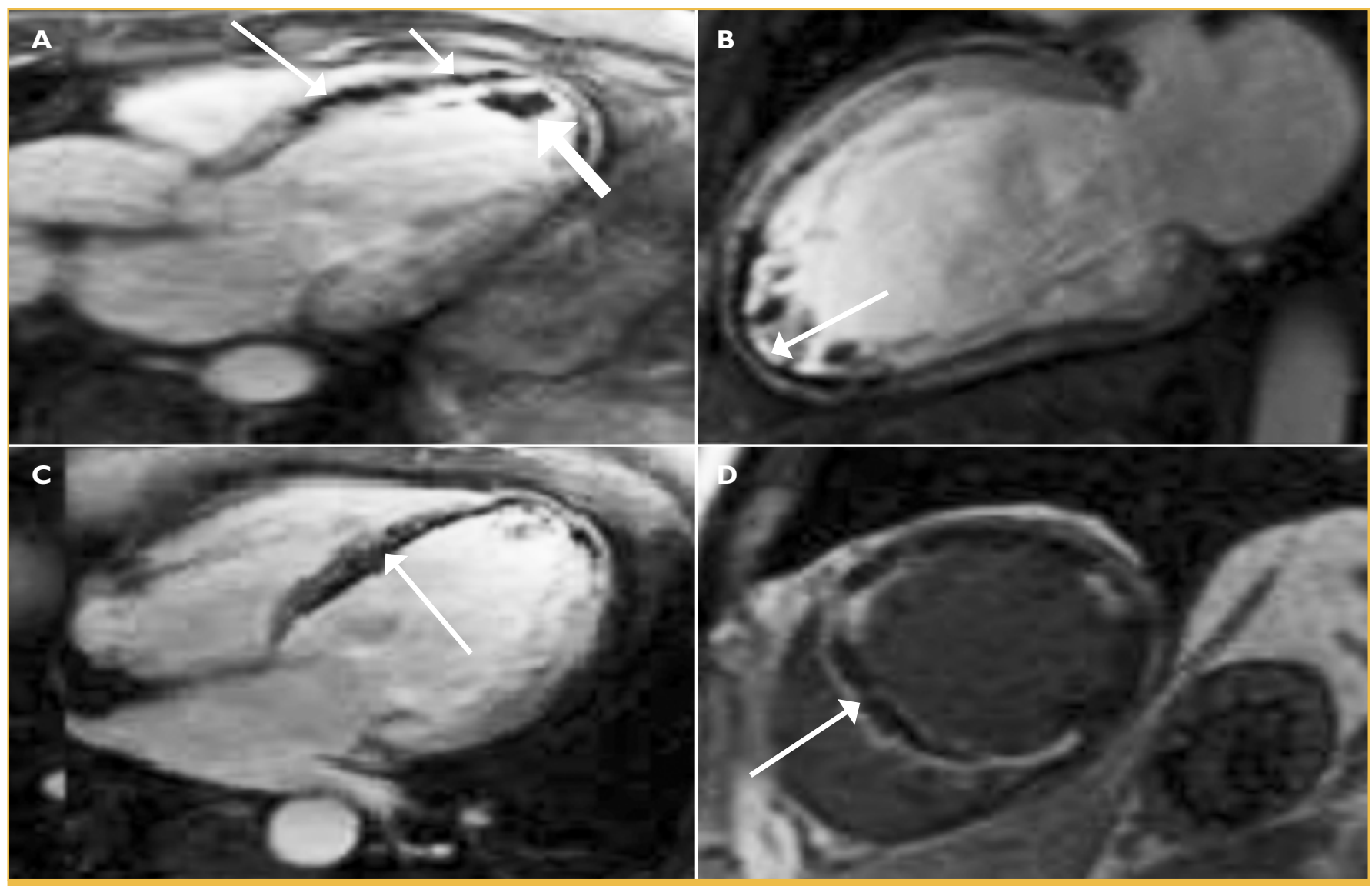

FIGURE 2: CMR in acute myocardial infarction.

Early and Late gadolinium imaging in the peri-infarct period.A, B \& C demonstrate microvascular obstruction (thin arrows) in anteroseptal and apical segments with LV thrombus (thick arrow). LGE in anteroseptal, inferoseptal and inferior segments in short axis.
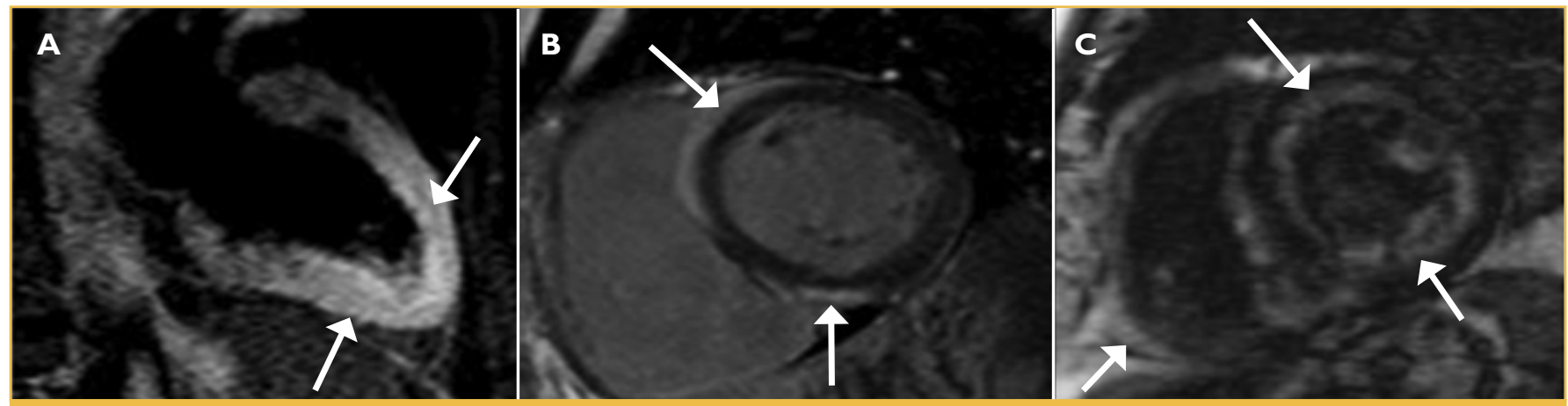

FIGURE 3: CMR in various Cardiomyopathies.

A. Takostubo Cardiomyopathy.T2-STIR imaging demonstrating increased signal intensity in mid to apical (arrows) LV indicating oedema. B. Cardiac Sarcoid. Patchy sub-epicardial hyperenhancement (arrows) in short axis view on late gadolinium imaging. C. Cardiac Amyloid. Global sub-endocardial hyperenhancement (arrows) of LV and RV in short axis view on late gadolinium imaging. 
employing DSE, ${ }^{(20)}$ have shown a linear relationship between the number of viable segments pre-revascularisation and change in LVEF post-revascularisation. Pegg, et al. have recently elegantly demonstrated that the sum of normal plus viable segments of greater than 10 on a 16 segment AHA model will accurately predict increase in LV ejection fraction. ${ }^{(21)}$

Fifty patients underwent CMR to assess LV function and viability before and 6 months after CABG. Viability was defined as transmural hyper-enhancement of $<50 \%$ (i.e. a binary variable) and the presence of 10 or more viable or normal segments predicted a gain of $3 \%$ in LVEF with a sensitivity and specificity of $95 \%$ and $75 \%$ respectively. Assessment of scar using LGE is undisputed in patients with either no LGE or $>75 \%$ transmurality. However, in intermediate transmural segments (25 to 75\%), low dose dobutamine to assess contractile reserve is considered superior to scar quantification.(22)

Kuhl, et al. studied 26 patients with ischaemic cardiomyopathy, with SPECT, ${ }^{(18)}$ F-flurodeoxyglucose Positron Emission Tomography (FDG PET) and LGE-CMR. LE-CMR was able to differentiate viable myocardium as defined by PET, with sensitivity of $96 \%$ and specificity of $84 \%$. This study demonstrated that LGE-CMR is as good as FDG PET in the assessment of myocardial viability. ${ }^{(23)}$ More recent literature shows that a combination of various CMR parameters such as wall thickness, scar quantification and contractile reserve are most efficient in predicting functional recovery. ${ }^{(24,25)}$

\section{Troponin positive chest pain}

Patients frequently present with troponin positive chest pain and are subsequently found to have patent coronary arteries at angiography. Previously these patients would have been permanently labelled as having had a myocardial infarction and treated as such. CMR has changed the paradigm of how these patients are managed. CMR performed during the acute presentation commonly reveals 3 possible aetiologies.

Firstly, it can show that acute myocarditis (often with no clear viral prodrome clinically) is present. A combined CMR approach using T2-weighted imaging and contrast enhanced TI weighted images, provides high diagnostic accuracy and is a useful tool in the diag- nosis and assessment of patients with suspected acute myocarditis. ${ }^{(26)}$ LGE in the setting of myocarditis has a "non-ischaemic" pattern, typically affecting the sub-epicardium and the mid-myocardial wall. This focal hyper-enhancement becomes diffuse over a period of days to weeks, then decreases during healing and may become invisible after recovery. Occasionally, the CMR reveals the diagnosis to be acute myocardial infarction even with relatively normal coronary arteries at angiography. Thirdly, CMR can show/ confirm the diagnosis of Stress (Takotsubo) cardiomyopathy. ${ }^{(27)}$ The characteristic patterns of oedema settle quickly in the above conditions and prompt imaging is recommended to increase diagnostic yield. Achieving an accurate diagnosis has obvious benefits for future management and life/health insurance purposes.

\section{Adult congenital heart disease}

Due to the flexibility and quality of the images obtainable, CMR is particularly well suited to imaging congenital heart disease. These patients often have poor echo windows and the right heart is notoriously difficult to assess fully. Serial follow up of great vessels and right heart dimensions is important when planning operative intervention and an accurate, non-ionising modality such as CMR is clearly preferable. Optimal visualisation of the right heart is important in the increasing recognised entity of arrhythmogenic right ventricular cardiomyopathy when wall motion and chamber size are part of the diagnostic criteria set out by ARVC Task Force. ${ }^{(28)}$

\section{Ischaemia assessment}

A significant percentage of CMR studies are dedicated to ischaemia testing. However, administration of dobutamine is suboptimal for patient comfort and care in the MR environment. The real advantage lies in first-pass myocardial perfusion imaging under vasodilator stress. Here, a bolus of gadolinium-based contrast agent is injected into a peripheral vein and a sequence of images is then obtained to follow the dynamic passage of dye through the heart. Vasodilatation with adenosine or dipyridamole induces an increase in blood flow (hyperaemia) in myocardial areas subtended by normal coronary arteries, whereas no or only minimal changes are found in areas supplied by stenotic coronary arteries. This relative hypo-enhancement in under perfused areas is usually evident visually and constitutes a perfusion defect (Figure 4). 
In a large, prospective study, Greenwood, et al. very elegantly demonstrated the superiority and high diagnostic accuracy of CMR over SPECT in the detection of coronary heart disease. ${ }^{(29)}$ They studied 752 patients with suspected coronary heart disease with multi-parametric CMR and Single-Photon Emission Computed Tomography (SPECT). X-ray coronary angiography was the reference standard. Multi-parametric CMR had a sensitivity of $86.5 \%$ (vs $66 \%$ ), specificity of $83 \%$ (vs $82 \%$ ) and negative predictive value of $90.5 \%$ (vs 79\%). Several single-centre studies and a published multicentre trial have compared perfusion CMR favourably (sensitivities $>90 \%$, specificity around 80 - 85\%) to existing nuclear methods (SPECT) or invasive cardiac catheterisation for the detection of myocardial ischaemia in the setting of chronic chest pain. (30) A negative CMR perfusion in patients presenting with chest pain without ECG changes or cardiac biomarker abnormality, have an excellent short to mid term prognosis. ${ }^{(31)}$

\section{WHEN SHOULD CMR NOT BE USED?}

CMR is not the panacea for all cardiac imaging requirements. Whilst perfusion imaging can quantify myocardial blood flow, a reliable, quick and robust luminographic assessment of the entire coronary arteries is not yet possible on most clinical systems. High-volume centres with appropriate skills can reliably image the proximal course of the coronary arteries to exclude anomalous coronary anatomy obviating the need for $\mathrm{CT}$ and the associated radiation.

The temporal resolution of CMR is currently limited which reduces the ability to detect small hypermobile structures such as vegetations. The sequelae of endocarditis such as root abscesses can be assessed however. ${ }^{(32)}$
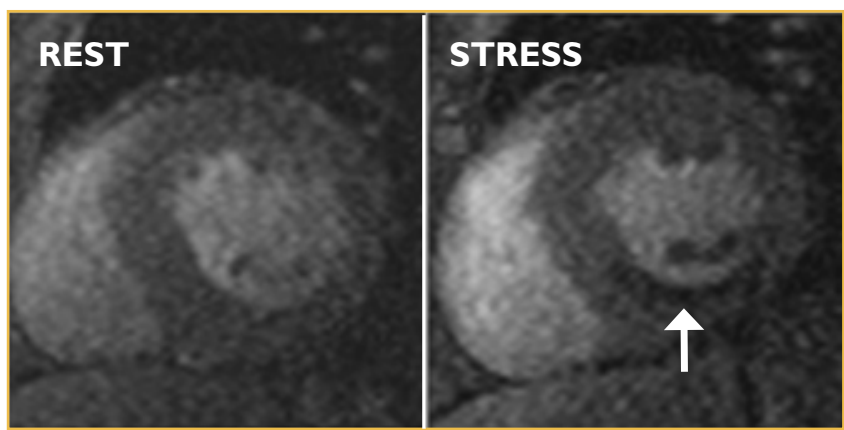

FIGURE 4: CMR-Stress perfusion imaging. Perfusion defect in the inferior segment (arrow).
Echocardiography remains supreme in the evaluation of valvular lesions however CMR can complement echo in cases where the degree of severity is unclear such as aortic regurgitation. Volumetric differences can be used to calculate regurgitant fractions in isolated valvular lesions and in combination with flow measurements in more complex cases. The same techniques can be used to assess shunting in congenital heart disease.

Finally, the multiple facets of CMR make the modality invaluable in the assessment of pericardial pathology and cardiac masses. Tumours can be reliably distinguished from other masses such as thrombus and often the type of tumour can be revealed by tissue characterisation sequences. ${ }^{(33)}$

\section{CONCLUSION}

Cardiac magnetic resonance imaging is a powerful clinical tool enabling cardiologists to accurately differentiate and diagnose cardiac pathology. CMR technology and applications continue to rapidly advance with positive implications for interventional cardiology and clinical research too. Useful resources for further reading can be found at www.scmr.org. The website has a vast and fascinating clinical case archive which will illustrate much of this article.

\section{Conflict of interest: none declared.}




\section{REFERENCES}

I. Bruder O, Schneider S, Nothnagel D, et al. EuroCMR (European Cardiovascular Magnetic Resonance) registry: Results of the German pilot phase. J Am Coll Cardiol 2009;54(15): 1457-66.

2. Cheng AS, Selvanayagam JB. High field cardiac magnetic resonance imaging current and future perspectives. Heart Lung Circ 20 I ; 19(3): 145-53.

3. Barkhausen J, Ruehm SG, Goyen M, et al. MR evaluation of ventricular function: True fast imaging with steady-state precession versus fast low-angle shot cine MR imaging: feasibility study. Radiology 2001;219(1):264-9.

4. Bellenger NG, Burgess MI, Ray SG, et al. Comparison of left ventricular ejection fraction and volumes in heart failure by echocardiography, radionuclide ventriculography and cardiovascular magnetic resonance: Are they interchangeable? Eur Heart J 2000;21 (16): 1387-96.

5. Pattynama PM, Lamb HJ, van der Velde EA, et al. Left ventricular measurements with cine and spin-echo MR imaging: A study of reproducibility with variance component analysis. Radiology 1993; 187(1):261-8.

6. Grothues F, Smith GC, Moon JC, et al. Comparison of interstudy reproducibility of cardiovascular magnetic resonance with two-dimensional echocardiography in normal subjects and in patients with heart failure or left ventricular hypertrophy. Am J Cardiol 2002;90(I):29-34

7. Grobner T. Gadolinium: A specific trigger for the development of nephrogenic fibrosing dermopathy and nephrogenic systemic fibrosis? Nephrol Dial Transplant 2006;2 I (4): I 104-8.

8. Marckmann P, Skov L, Rossen K, et al. Nephrogenic systemic fibrosis: Suspected causative role of gadodiamide used for contrast-enhanced magnetic resonance imaging. J Am Soc Nephrol 2006; 17(9):2359-62.

9. Broome DR, Girguis MS, Baron PW. Gadodiamide-associated nephrogenic systemic fibrosis: Why radiologists should be concerned. AJR Am J Roentgenol 2007; | 88(2):586-92.

10. Hunold P, Schlosser T, Vogt FM, et al. Myocardial late enhancement in contrastenhanced cardiac MRI: Distinction between infarction scar and non-infarctionrelated disease. AJR Am J Roentgenol 2005; I 84(5): 1 420-6.

I I. Porto I, Selvanayagam J, Ashar $V$, et al. Safety of magnetic resonance imaging I to 3 days after bare metal and drug-eluting stent implantation. Am J Cardio 2005;96(3):366-8.

12. Gerber TC, Fasseas P, Lennon RJ, et al. Clinical safety of magnetic resonance imaging early after coronary artery stent placement. J Am Coll Cardiol 2003; 42(7): 1295-8.

13. Moon JC, Fisher NG, McKenna WJ, et al. Detection of apical hypertrophic cardiomyopathy by cardiovascular magnetic resonance in patients with nondiagnostic echocardiography. Heart 2004;90(6):645-9.

14. McCrohon JA, Moon JC, Prasad SK, et al. Differentiation of heart failure related to dilated cardiomyopathy and coronary artery disease using gadolinium-enhanced cardiovascular magnetic resonance. Circulation 2003; I08(I):54-9.

15. Mahrholdt H, Wagner A, Judd RM, et al. Delayed enhancement cardiovascular magnetic resonance assessment of non-ischaemic cardiomyopathies. Eur Heart J 2005;26(I5): | 466|-74

16. Reimer KA, Jennings RB. The "wavefront phenomenon" of myocardial ischaemic cell death. II. Transmural progression of necrosis within the framework of ischaemic bed size (myocardium at risk) and collateral flow. Lab Invest 1979; 40(6):633-44.

17. Leong DP, Chakrabarty A, Shipp N, et al. Effects of myocardial fibrosis and ventricular dyssynchrony on response to therapy in new-presentation idiopathic dilated cardiomyopathy: Insights from cardiovascular magnetic resonance and echocardiography. Eur Heart J 2011 .

18. Selvanayagam JB, Kardos A, Francis JM, et al. Value of delayed-enhancement cardiovascular magnetic resonance imaging in predicting myocardial viability after surgical revascularisation. Circulation 2004; I I0(12): 1535-4I.
19. Kim RJ, Wu E, Rafael A, Chen EL, et al. The use of contrast-enhanced magnetic resonance imaging to identify reversible myocardial dysfunction. N Engl J Med 2000;343(20): 1 445-53.

20. Rizzello V, Poldermans D, Boersma E, et al. Opposite patterns of left ventricular remodeling after coronary revascularisation in patients with ischaemic cardiomyopathy: Role of myocardial viability. Circulation 2004; I 10(16):2383-8.

21. Pegg TJ, Selvanayagam JB, Jennifer J, et al. Prediction of global left ventricular functional recovery in patients with heart failure undergoing surgical revascularisation, based on late gadolinium enhancement cardiovascular magnetic resonance. J Cardiovasc Magn Reson 2010;12:56.

22. Wellnhofer E, Olariu A, Klein C, et al. Magnetic resonance low-dose dobutamine test is superior to SCAR quantification for the prediction of functional recovery. Circulation 2004;109(18):2172-4.

23. Kuhl HP, Beek AM, van der Weerdt AP, et al. Myocardial viability in chronic ischaemic heart disease: Comparison of contrast-enhanced magnetic resonance imaging with (18)F-fluorodeoxyglucose positron emission tomography. J Am Coll Cardiol 2003;4 I (8): I 341-8.

24. Glaveckaite S, Valeviciene N, Palionis D, et al. Value of scar imaging and inotropic reserve combination for the prediction of segmental and global left ventricular functional recovery after revascularisation. J Cardiovasc Magn Reson 20 I 1; 1 3:35.

25. Kirschbaum SW, Rossi A, Boersma E, et al. Combining magnetic resonance viability variables better predicts improvement of myocardial function prior to percutaneous coronary intervention. Int J Cardiol 201 I.

26. Abdel-Aty $\mathrm{H}$, Boye $\mathrm{P}$, et al. Diagnostic performance of cardiovascular magnetic resonance in patients with suspected acute myocarditis: Comparison of different approaches. J Am Coll Cardiol 2005;45(I I): I8I5-22.

27. Eitel I, von Knobelsdorff-Brenkenhoff F, Bernhardt P, et al. Clinical characteristics and cardiovascular magnetic resonance findings in stress (Takotsubo) cardiomyopathy. JAMA 201 I;306(3):277-86.

28. Marcus Fl, McKenna WJ, Sherrill D, et al. Diagnosis of arrhythmogenic right ventricular cardiomyopathy/dysplasia: Proposed modification of the task force criteria. Circulation 20 I0; 12 I (13): I533-4|.

29. Greenwood JP, Maredia N, Younger JF, et al. Cardiovascular magnetic resonance and single-photon emission computed tomography for diagnosis of coronary heart disease (CE-MARC): A prospective trial. Lancet 2012;379(98/4):453-60.

30. Schwitter J, Wacker CM, van Rossum AC, et al. MR-IMPACT: Comparison of perfusion-cardiac magnetic resonance with single-photon emission computed tomography for the detection of coronary artery disease in a multicentre, multivendor, randomised trial. Eur Heart J 2008;29(4):480-9.

31. Lerakis S, McLean DS, Anadiotis AV, et al. Prognostic value of adenosine stress cardiovascular magnetic resonance in patients with low-risk chest pain. J Cardiovasc Magn Reson 2009; 1 1:37.

32. Sverdlov AL, Taylor K, Elkington AG, et al. Images in cardiovascular medicine. Cardiac magnetic resonance imaging identifies the elusive perivalvular abcess. Circulation 2008; I /8(I):el-3.

33. Fussen S, De Boeck BW, Zellweger MJ, et al. Cardiovascular magnetic resonance imaging for diagnosis and clinical management of suspected cardiac masses and tumours. Eur Heart J 20 | 1;32( I 2): 1 55 | -60. 